\title{
Organ Polarity in Plants Is Specified through the Opposing Activity of Two Distinct Small Regulatory RNAs
}

\author{
F.T.S. Nogueira, ${ }^{*}$ A.K. Sarkar, ${ }^{*}$ D.H. Chitwood, ${ }^{* \dagger}$ and M.C.P. Timmermans* \\ *Cold Spring Harbor Laboratory, Cold Spring Harbor, New York 11724; \\ ${ }^{\dagger}$ Watson School of Biological Sciences, Cold Spring Harbor, New York 11724
}

\begin{abstract}
Small RNAs and their targets form complex regulatory networks that control cellular and developmental processes in multicellular organisms. In plants, dorsoventral (adaxial/abaxial) patterning provides a unique example of a developmental process in which early patterning decisions are determined by small RNAs. A gradient of microRNA166 on the abaxial/ventral side of the incipient leaf restricts the expression of adaxial/dorsal determinants. Another class of small RNAs, the TAS3-derivated trans-acting short-interfering RNAs (ta-siRNAs), are expressed adaxially and repress the activity of abaxial factors. Loss of maize leafbladeless 1 (lbll) function, a key component of the ta-siRNA biogenesis pathway, leads to misexpression of miR166 throughout the initiating leaf, implicating ta-siRNAs in the spatiotemporal regulation of miR166. The spatial restriction of tasiRNA biogenesis components suggests that this pathway may act non-cell-autonomously in the meristem and possibly contributes to the classic meristem-borne adaxializing Sussex signal. Here, we discuss the key participants in adaxial/abaxial patterning and point out the intriguing possibility that organ polarity in plants is established by the opposing action of specific ta-siRNAs and miRNAs.
\end{abstract}

Plant shoots are characterized by indeterminate growth. The growing tip of a plant, referred to as the shoot apical meristem (SAM), contains a population of pluripotent stem cells that divide to maintain the SAM and to generate daughter cells from which lateral organs, such as leaves, arise (Fig. 1). Leaves of most seed plants are dorsoventrally flattened and differentiate distinct cell types in the upper and lower leaf surfaces to maximize the capture of sunlight and the exchange of gases that drive photosynthesis. Dorsoventral (adaxial/abaxial) polarity is specified shortly after the emergence of the leaf primordium from the meristem and is thought to reflect inherent positional differences in the developing organ relative to the SAM (Wardlaw 1940). The adaxial, dorsal side of the leaf develops in closer proximity to the tip of the SAM than the abaxial, ventral side (Fig. 1) (for review, see Bowman et al. 2002). Surgical experiments separating the incipient leaf from the remainder of the SAM result in radially symmetric, abaxialized leaves suggesting the existence of a meristem-borne signal that specifies adaxial cell fate (Sussex 1951, 1955). Although the identity of the Sussex signal remains unknown, recent studies implicate small regulatory RNAs in adaxial/abaxial patterning and raise the possibility that a mobile RNA might fulfill the requirements of a positional, polarizing signal. Here, we review the role of microRNAs (miRNAs) and trans-acting short interfering RNAs (ta-siRNAs) in leaf polarity and outline recent results suggesting that the opposing activity of two distinct small regulatory RNAs establishes adaxial/abaxial asymmetry in the developing leaf.

\section{ESTABLISHMENT OF LEAF POLARITY IN ARABIDOPSIS}

Several families of putative transcription factors play key roles in establishing adaxial/abaxial polarity in the

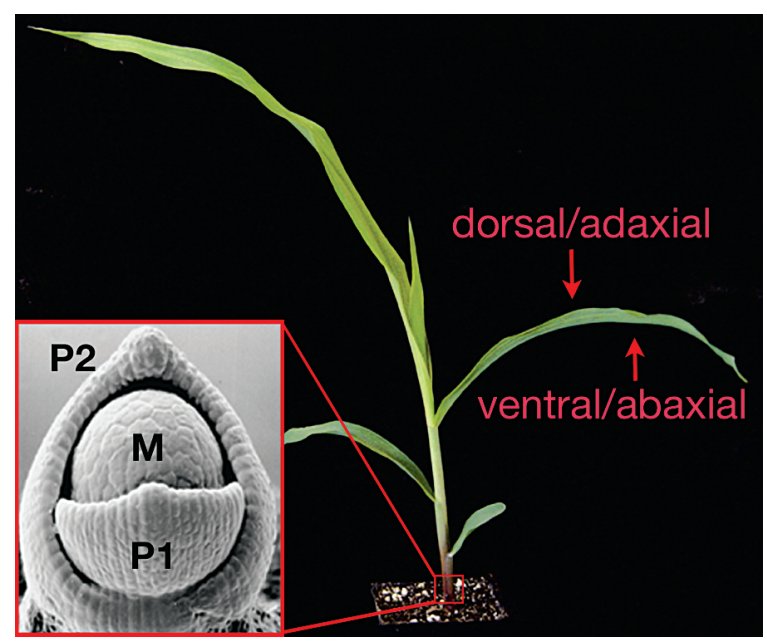

Figure 1. Leaf primordia arise on the flank of the shoot apical meristem (SAM) and establish dorsoventral (adaxial/abaxial) polarity in response to signals from the SAM. Leaves of a 2week-old maize seedling differentiate distinct dorsal (adaxial) and ventral (abaxial) surfaces. The red box indicates the approximate position of the SAM within the surrounding older leaves. The inset shows a scanning electron micrograph of a maize apex. The population of stem cells at the tip of the meristem (M) permits the reiterative development of the leaf primordia, which emerge from the flank of the SAM. The youngest leaf primordium is indicated as $\mathrm{P} 1$, the second youngest as $\mathrm{P} 2$, etc.

leaf. Members of the class III family of homeodomainleucine zipper (HD-ZIPIII) proteins-PHABULOSA (PHB), PHAVOLUTA (PHV), and REVOLUTA (REV) - specify adaxial fate (McConnell et al. 2001; Otsuga et al. 2001; Emery et al. 2003). In contrast, the KANADI $(K A N)$ genes, which encode transcriptional regulators belonging to the GARP family, act redundantly to 
promote abaxial identity (Eshed et al. 2001, 2004; Kerstetter et al. 2001). Although both the HD-ZIPIII and $K A N$ genes are expressed evenly throughout the incipient leaf (P0), their domains of expression become restricted to the adaxial and abaxial sides of the organ, respectively, shortly after emergence of the primordium from the SAM. Constitutive $K A N$ expression leads to the development of a radially symmetric, abaxialized leaf (Eshed et al. 2001, 2004; Kerstetter et al. 2001). Loss of HD-ZIPIII function results in a similar phenotype, suggesting that the $H D$ ZIPIII genes act, at least in part, to spatially restrict the $K A N$ expression domain (Emery et al. 2003). HD-ZIPIII expression is in turn excluded from the abaxial side by the action of the KAN proteins. Thus, the HD-ZIPIII and $K A N$ genes have a mutually antagonistic relationship, which may reflect their requirement to maintain a stable adaxial/abaxial boundary throughout leaf development.

In Arabidopsis, establishment of abaxial identity further requires the activities of members of the $Y A B B Y$ and AUXIN RESPONSE FACTOR (ARF) families (Sawa et al. 1999; Siegfried et al. 1999; Pekker et al. 2005). The YABBY genes FILAMENTOUS FLOWER (FIL) and $Y A B 3$ act, at least in part, downstream of the HD-ZIPIII and $K A N$ genes, whereas $A R F 3 / E T T$ and $A R F 4$ affect organ polarity through a distinct pathway (Sawa et al. 1999; Siegfried et al. 1999; Kumaran et al. 2002; Pekker et al. 2005). ARF3/ETT is expressed more broadly than $A R F 4$, but both transcription factors colocalize in the abaxial domain of leaf primordia, where they act in combination with KAN proteins to promote abaxial fate (Pekker et al. 2005).

Interestingly, both the abaxial determinants $A R F 3 / E T T$ and $A R F 4$, as well as the adaxializing $H D$-ZIPIII genes, are targets for RNAi-based regulation (Fig. 2a). HDZIPIII transcripts contain complementary target sites to the nearly identical microRNAs 165 and 166 (miR165/166), which can direct cleavage of HD-ZIPIII mRNAs in vitro (Rhoades et al. 2002; Tang et al. 2003). ARF3/ETT and ARF4 are targets of the recently discovered ta-siRNAs (Allen et al. 2005; Williams et al. 2005a). Thus, in addition to being a key developmental process, the prominent role of small regulatory RNAs in adaxial/abaxial patterning makes leaf polarity an excellent model to dissect the role of small RNAs as developmental signals.

\section{BIOGENESIS AND FUNCTION OF MIRNAS}

The biogenesis and function of plant miRNAs have been extensively reviewed elsewhere (see Timmermans et al. 2004; Jones-Rhoades et al. 2006; Mallory and Vaucheret 2006). Briefly, miRNAs are processed from

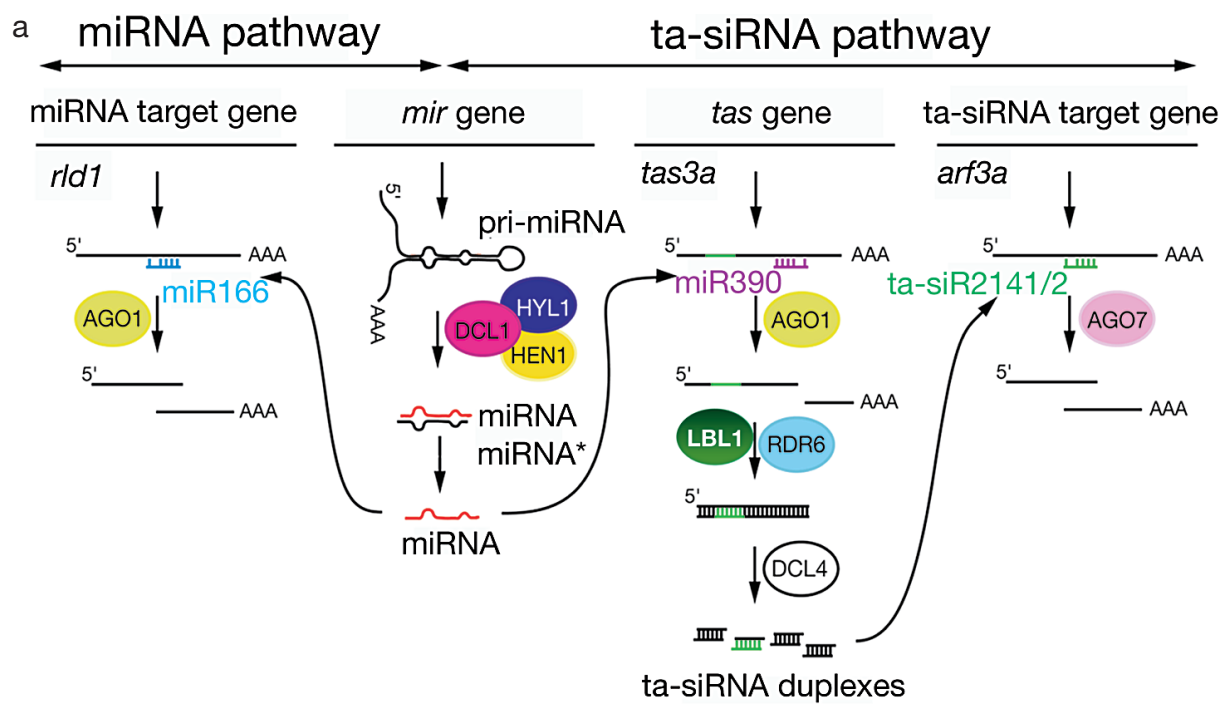

b

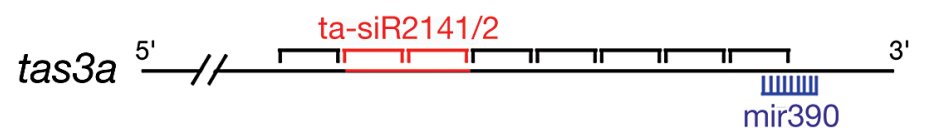

Figure 2. Plant miRNA and ta-siRNA biogenesis and function are interconnected. (a) mir genes transcribe a primary miRNA transcript (pri-miRNA), which is rapidly processed by DCL1 and HYL1. The resulting imperfect miRNA/miRNA* duplex is $3^{\prime}$-end methylated by HEN1 and loaded onto an AGO1-containing RISC. This complex cleaves target transcripts; HD-ZIPIII (rld1) transcripts in case of miR166 and tas $3 a$ mRNAs for miR390. The tas $3 a$ cleavage fragments are converted into double-stranded RNAs through the activities of LBL1/SGS3 and RDR6, and processed by DCL4 into 21-nucleotide double-stranded siRNAs. The tas3aderived ta-siR2141/2142 acts in trans to cleave arf3a transcripts via AGO7. (b) Diagram of the maize tas $3 a$ transcript illustrating the 21-nucleotide phased processing of ta-siRNAs, which initiates from the miR390-cleavage site. Black brackets represent putative tasiRNAs, and red brackets correspond to Arabidopsis ta-siR2141/ta-siR2142 siRNA homologs. 
RNA polymerase II transcripts that contain a stem-loop structure (Fig. 2a). These transcripts, termed primary miRNAs (pri-miRNAs), are processed by the RNase III enzyme DICER-LIKE1 (DCL1) (Park et al. 2002; Reinhart et al. 2002). Subsequently, the approximately 21-nucleotide mature miRNA becomes incorporated into the RNA-induced silencing complex (RISC), which targets complementary transcripts for site-specific cleavage or translational repression (Chen 2004; Han et al. 2004; Vaucheret et al. 2004; Baumberger and Baulcombe 2005). Plant miRNAs and their targets frequently possess near-perfect complementarity, and this has enabled the identification of many target genes using computational approaches. Interestingly, the known plant miRNAs show a strong propensity to target transcription factors or other genes that regulate critical steps during plant development (Reinhart et al. 2002; Jones-Rhoades et al. 2006). Consequently, mutations in genes associated with miRNA biogenesis or function, such as ARGONAUTE1 (AGO1), HYPONASTIC LEAVES1 (HYL1), and SERRATE (SE), affect important developmental processes including adaxial/abaxial patterning (Kidner and Martienssen 2004; Grigg et al. 2005; Yu et al. 2005; Yang et al. 2006).

Elucidation of the precise developmental roles of individual miRNAs is, however, complicated by the presence of extensive redundancy. Most MIR genes are members of multigene families (see Bartel 2004; Jones-Rhoades et al. 2006). Whereas miRNA families in animals are small and include diverse members, plant miRNA families frequently contain many genes that can produce identical mature miRNA sequences. Family members are likely to have overlapping expression profiles and functions that buffer against the loss of any single miRNA locus, as few loss-offunction alleles of MIR genes have been recovered through forward-genetic screens. Dominant gain-of-function miRNA mutants, however, are more common and have revealed developmental roles for several miRNAs. For example, the meristem enlarged 1 and jabbal-D mutants both develop fasciated stems and enlarged meristems resulting from altered MIR166a and MIR166g expression, respectively (Kim et al. 2005; Williams et al. 2005b). Similar gain-of-function mutations for miR156, miR159, miR160, miR164, miR172, and miR319 lead to defects in vegetative and floral organ development, meristem function, and flowering (see Jones-Rhoades et al. 2006).

\section{ROLE OF MIRNA165/166 IN ADAXIAL/ABAXIAL PATTERNING}

As in Arabidopsis, adaxial/abaxial asymmetry in maize is established through the polarized expression of $H D$ ZIPIII genes (Juarez et al. 2004b). rolled leafl (rld1), which encodes a close homolog of $R E V$, is expressed at the tip of the SAM and in a strip of cells from the center of the SAM to the site of leaf initiation (Fig. 3e). In developing leaf primordia, $r l d 1$ is expressed on the adaxial side as well as in the vasculature. The miR165/166 target site in the HD-ZIPIII transcripts is conserved between monocots and dicots (Reinhart et al. 2002; Rhoades et al. 2002; Juarez et al. 2004b). Because these lineages last shared a
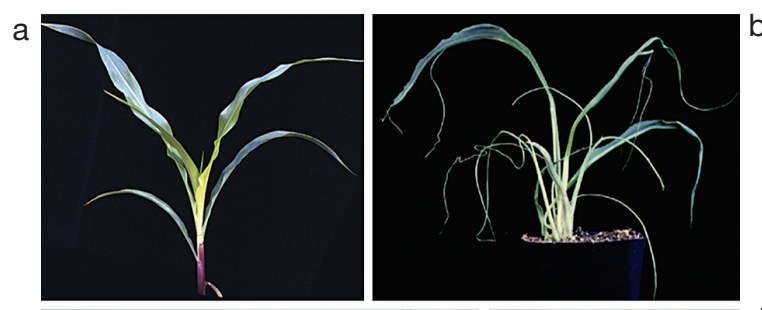

C
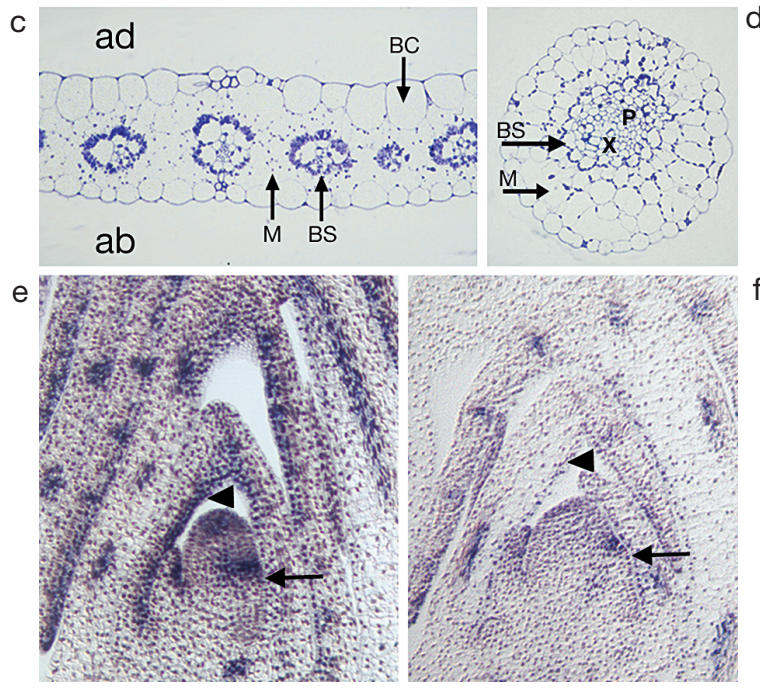

Figure 3. LBL1 is required for the specification of adaxial/dorsal fate. Compared to the wild-type maize seedling $(a)$, severe lbll mutants (b) develop thread-like, abaxialized leaves. (c) Transverse section through a wild-type leaf blade illustrates its dorsoventrally flattened organization, with polar veins surrounded by bundle sheath (BS) and mesophyll cells (M). Bulliform cells (BC) differentiate only in the adaxial (ad) epidermis. (d) Severe $l b l l$ leaves are radially symmetric and comprise an irregular vascular cylinder surrounded by concentric rings of bundle sheath and mesophyll cells, and abaxial (ab) epidermis. X, xylem; P, phloem. (e) Longitudinal section through a wild-type apex showing rldl expression in the SAM, vasculature, and on the adaxial side of the incipient (arrow) and developing leaf primordia (arrowhead). (f) In $l b l l$, meristematic and adaxial expression of $r l d 1$ is reduced. (Reprinted, with permission, from Timmermans et al. 1998 and Juarez et al. 2004a.)

common ancestor more than 100 million years ago, this conservation suggests an important role in plant development for the regulation of HD-ZIPIII genes by miR165/166. The significance of this relationship was first demonstrated by the characterization of dominant mutations in the Arabidopsis HD-ZIPIII genes ( $p h b-d$, $p h v-d$, and $r e v-d$ ) that abrogate the miR165/166 target site. Such mutations interfere with miRNA-directed transcript cleavage, leading to ectopic, abaxial HD-ZIPIII expression and formation of adaxialized leaves (McConnell et al. 2001; Emery et al. 2003; Tang et al. 2003). Disruption of the miRNA165/166 complementary site of maize rld1 (Rld1-O) similarly results in misexpression of $r l d 1$ on the abaxial side of developing primordia and adaxialization of the leaf (Juarez et al. 2004b).

In situ hybridization provides direct evidence that the pattern of miR165/166 expression spatially defines the expression domain of the HD-ZIPIII genes (Juarez et al. 2004b; Kidner and Martienssen 2004). miR165 and 
miR166 are expressed on the abaxial side of the developing leaf in a pattern complementary to that of the $H D$ ZIPIII genes. Interestingly, in maize, miR166 is most abundant in a group of cells below the incipient leaf, but a gradient of weaker miR166 expression extends into the abaxial side of the newly initiating primordium. In P1 primordia, higher levels of miR166 accumulate abaxially and, in older primordia, miR166 accumulates in a progressively broader domain extending adaxially and laterally (Juarez et al. 2004b). These findings suggest that miR165/166 is a highly conserved polarizing signal that specifies adaxial/abaxial polarity by restricting $H D$ ZIPIII expression to the adaxial side of the leaf in both dicots and monocots (Timmermans et al. 2004). The spatial regulation of $H D-Z I P I I I$ gene expression by miR165/166 may even predate the origin of angiosperm leaves. The miR165/166-directed cleavage of HD-ZIPIII transcripts is conserved in basal lineages of land plants, including bryophytes, lycopods, and ferns (Floyd and Bowman 2004). Organ polarity is a relatively recent landmark in plant development; thus, it is possible that the regulation of HD-ZIPIII genes by miR165/166 evolved as a preadaptation that was later co-opted for use in adaxial/abaxial patterning of leaves and other lateral organs (Floyd et al. 2006).

\section{THE TA-SIRNA PATHWAY}

Like the miRNAs, ta-siRNAs are processed from long, RNA pol II transcripts that are not predicted to encode for proteins (Fig. 2a). Interestingly, the ta-siRNA precursors $(T A S)$ are themselves targets for miRNA-directed cleavage (Allen et al. 2005). However, unlike most miRNAdirected cleavage products, TAS cleavage fragments are converted into double-stranded RNAs through the activities of the plant-specific Zn-finger protein SUPPRESSOR OF GENE SILENCING3 (SGS3) and RNA-DEPENDENT RNA POLYMERASE6 (RDR6), and are subsequently processed by DCL4 into 21-bp siRNAs that guide the cleavage of target mRNAs, similar to the action of miRNAs (Peragine et al. 2004; Vazquez et al. 2004; Allen et al. 2005; Gasciolli et al. 2005; Xie et al. 2005; Yoshikawa et al. 2005). Biogenesis of ta-siRNAs thus requires the activity of proteins in the miRNA pathway, such as DCL1 and AGO1, as well as SGS3, RDR6, and DCL4 (Fig. 2a).

Three gene families are known to generate ta-siRNAs in Arabidopsis. TAS1 and TAS2 are targets of miR173, whereas the production of ta-siRNAs from TAS3 depends on miR390-mediated cleavage (Allen et al. 2005). Because DCL4 cleavage initiates at the processed end of the TAS precursor, ta-siRNAs are generated in a 21nucleotide phase starting at the miRNA cleavage site (Fig. 2b). Consequently, the ta-siRNAs derived from each $T A S$ locus, as well as their potential targets, can be predicted using computational approaches. The TAS1/TAS2 loci share homology and produce related ta-siRNAs directed against a subset of pentatricopeptide repeat genes and a group of genes of unknown function (Peragine et al. 2004; Vazquez et al. 2004; Allen et al. 2005; Yoshikawa et al. 2005). Mutational analysis indicates that the TAS1- and
TAS2-derived ta-siRNAs have no obvious developmental role (Adenot et al. 2006). In contrast, two of the TAS3derived ta-siRNAs, ta-siR2141/2, regulate the expression of $A R F 2, A R F 3 / E T T$, and $A R F 4$, which are known to function during shoot morphogenesis and adaxial/abaxial patterning (Allen et al. 2005; Pekker et al. 2005; Williams et al. 2005a).

\section{TAS3 TA-SIRNAS SPECIFY LEAF POLARITY THROUGH REGULATION OF MIR166}

Given that the abaxial determinants ARF3/ETT and $A R F 4$ are targets for TAS3 ta-siRNAs, a role for the tasiRNA pathway in leaf polarity can be inferred. However, the contribution of this pathway to adaxial/abaxial patterning in Arabidopsis is not immediately apparent. Arabidopsis mutants that block the biogenesis of tasiRNAs develop no obvious leaf polarity defects (Peragine et al. 2004; Vazquez et al. 2004; Allen et al. 2005; Adenot et al. 2006). Moreover, the distribution of trichomes on leaves of such mutants, as well on plants expressing a ta-siR2141/2 insensitive allele of $A R F 3 / E T T$, is inconsistent with the predicted abaxializing phenotype (Peragine et al. 2004; Fahlgren et al. 2006; Hunter et al. 2006).

An essential role for the ta-siRNA pathway in adaxial/abaxial patterning became evident through the cloning and characterization of leafbladeless 1 (lbll) from maize. $l b l l$, in addition to the HD-ZIPIII genes, is required for the specification of adaxial fate (Timmermans et al. 1998; Juarez et al. 2004a). Loss-offunction $l b l 1$ mutations condition an abaxialized leaf phenotype, with the most severely affected $l b l l$ leaves becoming radially symmetric and fully abaxialized (Fig. 3a-d) (Timmermans et al. 1998). The recent cloning of lbl1 showed that it encodes a homolog of the Arabidopsis SGS3 protein. LBL1 and the Arabidopsis SGS3 protein share $65 \%$ amino acid similarity overall, but the degree of sequence similarity is higher in the zinc-finger (92\%) and XS domains (79\%), which define the SGS3 protein family (Fig. 4) (Bateman 2002).

These findings indicate that SGS3 activity is essential for adaxial/abaxial patterning in maize, and suggest a role for ta-siRNAs in this process. $\operatorname{miR} 173$ and the TAS1/TAS2 loci are not conserved between maize and Arabidopsis, but the maize genome includes at least one mir390 gene and four tas3 loci (tas $3 a-\operatorname{tas} 3 d)$ whose transcripts are predicted targets for miR390. Most tas3derived ta-siRNAs are not conserved between maize and Arabidopsis, but interestingly, all four maize tas 3 transcripts are predicted to yield copies of ta-siR2141/2 (Fig. b). The tas 3 genes, as well as mir390, are expressed in vegetative apices, and accordingly, ta-siR2141/2 accumulates in this tissue (F. Nogueira et al., in prep.). tasiR2141/2 accumulation is severely reduced or abolished in $l b l l$ mutants, suggestive of functional conservation between LBL1 and SGS3. As in Arabidopsis, of the maize tas3-derived ta-siRNAs, only ta-siR2141/2 has clearly identifiable candidate targets, and these include members of the arf3 gene family (F. Nogueira et al., in prep.). 5' RACE analysis identified $\operatorname{arf} 3 a$ as a direct tar- 

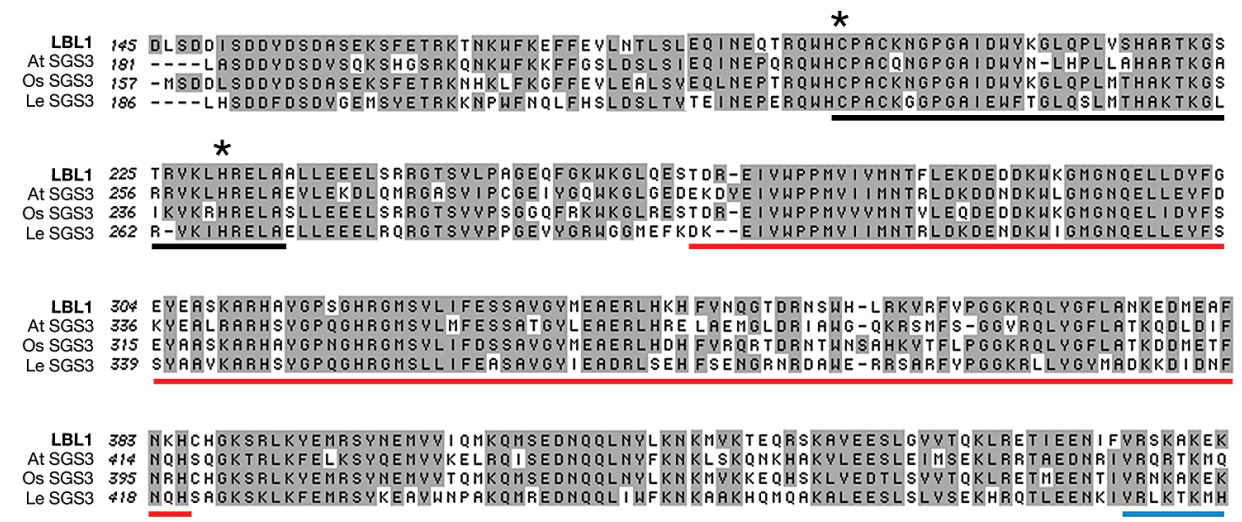

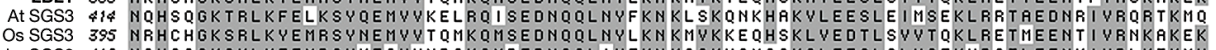
LESGS3 418 MQHSAGKSKLKFEMRSYKEAUWNPAKQMREDNQQL I WFKNKAGKHQMQAKALEESLSLVSEKHRQTLEENKIVRLKTKMH

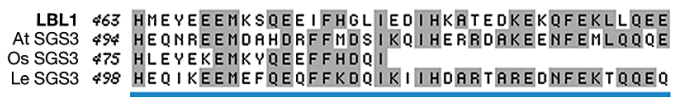

Figure 4. $l b l 1$ encodes a SGS3-like protein. Partial sequencing alignment of LBL1 (DQ832257) and the SGS3 proteins from Arabidopsis thaliana (NP_197747.1), Oryza sativa (AK064217.1), and Lycopersicum esculentum (BT013417.1). The zinc-finger domain (black), XS domain (red), and coiled coils (blue) characteristic of the SGS3 protein family are underlined. Asterisks mark amino acid substitutions identified in severe $l b l l$ alleles.

get of ta-siR2141/2, indicating that the ta-siRNA pathway is extensively conserved between maize and Arabidopsis. This pathway could be even more ancient, because homologs of miR390 and TAS3 have been identified in moss, which last shared a common ancestor with the monocots and dicots more than 400 million years ago (Arazi et al. 2005; D. Bartel, pers. comm.). Importantly, lbll is required for the ta-siR2141/2-directed cleavage of $\operatorname{arf} 3 a$, and thus forms an essential component of the tasiRNA pathway. The observation that LBL1 is essential for adaxial/abaxial patterning in maize thus implies a key role for ta-siRNAs in this process.

lbll contributes to leaf polarity by regulating the expression of HD-ZIPIII genes (Juarez et al. 2004a; F. Nogueira et al., in prep.). Expression of rldl and its paralogs $r l d 2$ and $p h b$ on the adaxial side of developing leaf primordia is reduced in $l b l l$ mutants (Fig. 3e,f). Notably, $H D$-ZIPIII expression at the side of leaf initiation is altered in $l b l l$ mutants, suggesting that $l b l l$ and the tasiRNA pathway affect an early step in adaxial/abaxial patterning. In situ hybridization confirms that $l b l l$ is most prominently expressed in a dome of cells at the tip of the SAM that extends into the adaxial side of the initiating primordium.

Loss of LBL1 activity leads to misexpression and/or overexpression of ta-siRNA targets and downstream components. Reduced HD-ZIPIII expression in $l b l l$, therefore, suggests that one or more antagonists of the $H D$-ZIPIII genes are controlled by the ta-siRNA pathway. Indeed, lbll affects $H D$-ZIPIII expression by regulating the spatiotemporal pattern of miR166 accumulation (F. Nogueira et al., in prep.). miR166 normally accumulates in a graded pattern on the abaxial side of incipient and young leaf primordia. However, in an $l b l 1$ mutant background, miR166 is ectopically expressed in a torus at the base of the SAM that broadly overlaps with the incipient leaf. Expression of miR166 in the P1 and older leaf pri- mordia also comprises a broader domain, including both adaxial and abaxial sides. Thus, $l b l l$ and the ta-siRNA pathway spatially restrict the miR166 expression domain in the SAM and developing leaf primordia, and contribute to organ polarity by setting up the abaxial specific expression gradient of miR166 in the incipient leaf. This presents the intriguing possibility that the opposing activity of two distinct small regulatory RNAs directs the early patterning decision leading to adaxial/abaxial polarity in the incipient leaf; ta-siR2141/2 defines the adaxial side of the leaf by restricting the expression domain of miR166, which in turn delineates the abaxial side by restricting expression of the adaxializing HD-ZIPIII genes.

The maize genome includes at least nine mir166 loci, mir166a through mir166i. All mir166 genes are expressed within the vegetative apex, but the spatiotemporal expression profiles vary among family members (F. Nogueira et al., unpubl.). The ta-siRNA pathway affects the accumulation of just a subset of mir166 precursors. Transcript levels for mir166c and mir 166i are increased in the meri-stem of $l b l l$ as compared to wild type, whereas mirl66a precursor levels are reduced in the mutant (F. Nogueira et al., in prep.). This suggests that the loss of ta-siRNA- directed repression of mir $166 \mathrm{c}$ and mir166i underlies, at least partially, the ectopic miR166 accumulation in $l b l l$ incipient primordia and resulting abaxialization of $l b l l$ leaves.

How might the ta-siRNA pathway restrict miR166 expression? Despite our increasing understanding of miRNA biogenesis and function, little is known about the regulation of MIR genes themselves. ARF proteins are transcription factors that mediate auxin-dependent gene regulation through binding to specific sequence motifs within promoters of auxin-regulated genes (Ulmasov et al. 1999). Although it will require gain- or loss-of-function arf3 mutants to assess the contribution of these transcription factors to the spatiotemporal regulation of miR166, it is not dif- 
ficult to envision that the ta-siRNA pathway may control the expression of specific mir166 family members via arf3 genes. Additionally, miRNA accumulation is likely regulated at the posttranscriptional level (Bollman et al. 2003; Yang et al. 2006). The primary mir166i transcript includes a sequence motif with modest complementarity to ta$\operatorname{siR} 2141 / 2$. Given that the ta-siRNA pathway is expected to occur in the nucleus (Vaucheret 2006), this observation suggests that the spatiotemporal pattern of miR166 expression may be regulated directly by this ta-siRNA. This possibility is particularly intriguing because it presents a scenario in which miRNA precursors are themselves controlled by small RNAs. Moreover, leaf polarity would be specified through a cascade of direct small regulatory RNA interactions in which miR390-directed cleavage of TAS3 triggers the production of ta-siR2141/2, which in turn processes mir166i required for the polarized expression of miR166 and adaxial/abaxial patterning of the newly initiated leaf.

\section{DIVERSE CONTRIBUTIONS OF THE TA-SIRNA PATHWAY TO LEAF POLARITY IN DISTINCT PLANT LINEAGES}

Despite uncertainty regarding the precise mechanism by which the ta-siRNA pathway regulates miR 166 expression, the defects observed in $l b l l$ clearly demonstrate a critical role for ta-siRNAs in adaxial/abaxial patterning by establishing the abaxial expression gradient of miR166 in the incipient leaf. These findings also highlight important differences between Arabidopsis and maize; namely, even though the ta-siRNA pathway is extensively conserved between these species, Arabidopsis mutants that block ta-siRNA biogenesis display no obvious leaf polarity defects (Peragine et al. 2004; Vazquez et al. 2004; Allen et al. 2005). The differential reliance on the ta-siRNA pathway for adaxial/abaxial patterning in part reflects redundancy of the pathway in Arabidopsis with ASYMMETRIC LEAVES1 (AS1) and AS2. Double mutants affecting the ta-siRNA pathway as well as $A S 1$ or $A S 2$ function develop weakly abaxialized leaves (Li et al. 2005; Garcia et al. 2006; Xu et al. 2006).

In addition, divergence in the nature or function of downstream targets, or the time during leaf development at which downstream targets act, could greatly influence the contribution of the ta-siRNA pathway to adaxial/abaxial patterning in different plant species. For instance, whereas the Arabidopsis ta-siRNA pathway represses FIL expression ( $\mathrm{Li}$ et al. 2005; Garcia et al. 2006; Xu et al. 2006), members of the maize yabby gene family closely related to FIL are positively regulated by lbll (Juarez et al. 2004a). Additionally, although expression analysis suggests that ta-siRNAs contribute to the regulation of miR165/166 in Arabidopsis (Li et al. 2005; $\mathrm{Xu}$ et al. 2006), disruption of the ta-siRNA pathway affects expression of specific MIR165/166 family members only during later stages of leaf development (F. Nogueira et al., unpubl.). Distinctly, the maize ta-siRNA pathway acts foremost in the SAM to regulate miR166 expression in the newly initiated primordium, and thus directs the early decisions in adaxial/abaxial patterning (F. Nogueira et al., in prep.).

\section{SMALL RNAS AS POTENTIAL MOBILE SIGNALS IN PLANTS}

The antagonistic interaction between ta-siRNAs and miR166 highlights the complexity that can be found in small RNA regulated pathways, and illustrates the important role of small RNAs in pattern formation during development. This unique interaction also raises the question whether small RNAs contribute to the production or perception of positional information from the SAM required for adaxial/abaxial patterning. Due to their intrinsic high specificity, the idea of mobilepossibly morphogenic-small RNA signals in plants is tantalizing. However, evidence so far suggests that miRNAs act largely cell-autonomously (Parizotto et al. 2004; Alvarez et al. 2006). siRNAs generated during posttranscriptional or virus-induced gene silencing, on the other hand, are mobile, and their movement is the basis for the non-cell-autonomous nature of these silencing processes (Himber et al. 2003; Voinnet 2005). Interestingly, cell-to-cell movement is restricted to 21bp DCL4-dependent siRNAs, whereas the 24-bp siRNAs generated by DCL3 act strictly cellautonomously outside the phloem (Dunoyer et al. 2005). This suggests that, if produced by the correct mechanism or channeled into the correct pathway, small RNAs can traffic between cells.

In this regard, the involvement of ta-siRNAs in the specification of adaxial fate is particularly intriguing. Their biogenesis requires DCL4, presenting the possibility that this novel class of siRNAs may be able to move from cell to cell. Indeed, ectopic expression of miR166 in the $l b l l$ mutant also occurs outside the normal $l b l 1$ expression domain. This nonoverlapping expression pattern, along with a role for ta-siRNAs in establishing a gradient of miR166 on the abaxial side of the incipient leaf, suggests that the ta-siRNA pathway may operate noncell-autonomously in the SAM (F. Nogueira et al., in prep.). ta-siRNAs thus constitute a plausible component of the Sussex signal. Consistent with this notion, the most severely affected $l b l l$ mutant leaves resemble the abaxialized leaves that arise following the surgical separation of leaf initials from the tip of the SAM (Fig. 3d) (Sussex 1951; Timmermans et al. 1998).

\section{CONCLUSIONS}

The finding that $l b l l$ encodes a key component of the ta-siRNA pathway reveals an essential role for tasiRNAs in adaxial/abaxial patterning. In addition, leaf polarity requires the activity of miR165/166. The importance of small regulatory RNAs in adaxial/abaxial polarity may reflect the need to rapidly change transcription profiles that underlie cell fate changes, or to maintain a precise balance between adaxial and abaxial determinants during primordium growth, but could also reflect a role of small RNAs acting as positional cues. The expanded uniform expression of miR166 in incipient primordia of $l b l l$ indicates that ta-siRNAs promote adaxial fate by restricting miR166 expression to the abaxial side of the initiating leaf. Organ polarity in 


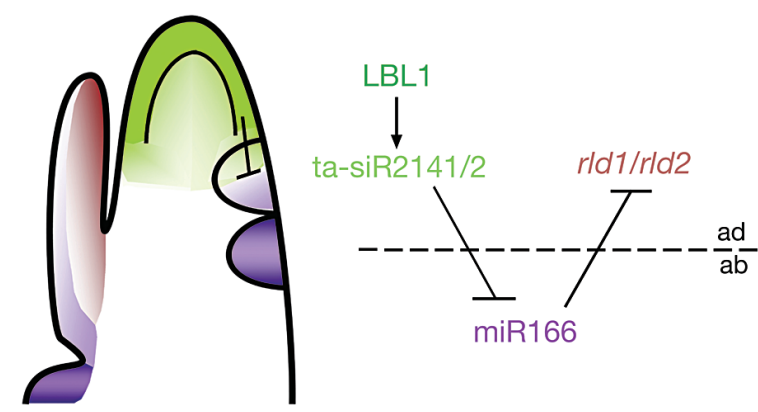

Figure 5. Organ polarity may be specified through the opposing activity of two distinct small regulatory RNAs. lbl1 (solid green) is expressed in a dome of cells at the tip of the SAM, suggesting the site of TAS3 ta-siRNA biogenesis. miR166 (purple) accumulates most prominently below the incipient leaf, but also in a graded pattern on the abaxial side of this primordium. tasiR2141/2 (pale green) may move from its side of synthesis into the adaxial side of the initiating leaf. ta-siR2141/2 locally restricts miR166 expression, which in turn represses expression of the adaxial determinants $r l d l$ and $r l d 2$ (red). The opposing activities of ta-siR2141/2 and miR166 thus set up polarity in the developing leaves.

plants may thus be specified through the opposing activities of ta-siR2141/2 and miR166 (Fig. 5).

The possibility that ta-siRNAs can act as mobile signals, perhaps because of their specific association with DCL4, suggests a mechanism by which ta-siR $2141 / 2$ establishes organ polarity. The local biogenesis of tasiR2141/2 in the SAM followed by its movement through the adjacent tissue would result in a concentration gradient across the initiating leaf, which sets up a complementary gradient of miR166. A defined balance between ta-siR2141/2 and miR166 activities may specify leaf polarity. Such a balance may result through regulated accumulation of each small RNA or through their relative efficacies of cleaving target transcripts. The number of cells over which a siRNA can move is correlated directly with its abundance, suggesting a dilution of the siRNA signal away from its point source (Himber et al. 2003; Voinnet 2005). The relatively low abundance of tasiR2141/2 (Allen et al. 2005; Williams et al. 2005a) or the involvement of an AGO7-based RISC in the TAS3 tasiRNA pathway could therefore be extremely significant for proper adaxial/abaxial patterning.

Although the concept of the Sussex signal has existed for more than 50 years, only recently have we started to better understand the complex genetic network that specifies adaxial/abaxial polarity. The recognition of small RNAs as early regulators of lateral organ polarity implicates them as potential polarizing signals that could perhaps contribute to the Sussex signal.

\section{ACKNOWLEDGMENTS}

The authors thank Shahinez Madi, Krista Marran, and Amanda Grieco for excellent technical assistance. This research was supported by grants from the U.S. Department of Agriculture (06-03420) and the National Science Foundation (0615752) to M.T.; F.N. was funded in part by a fellowship from the Cold Spring Harbor Laboratory Association; and D.C. is a National Science Foundation graduate research fellow and a George A. and Marjorie H. Matheson fellow.

\section{REFERENCES}

Adenot X., Elmayan T., Lauressergues D., Boutet S., Bouche N., Gasciolli V., and Vaucheret H. 2006. DRB4-dependent TAS3 trans-acting siRNAs control leaf morphology through AGO7. Curr. Biol. 16: 927.

Allen E., Xie Z., Gustafson A.M., and Carrington J.C. 2005. microRNA-directed phasing during trans-acting siRNA biogenesis in plants. Cell 121: 207.

Alvarez J.P., Pekker I., Goldshmidt A., Blum E., Amsellem Z., and Eshed Y. 2006. Endogenous and synthetic microRNAs stimulate simultaneous, efficient, and localized regulation of multiple targets in diverse species. Plant Cell 18: 1134.

Arazi T., Talmor-Neiman M., Stav R., Riese M., Huijser P., and Baulcombe D.C. 2005. Cloning and characterization of micro-RNAs from moss. Plant J. 43: 837.

Bartel D.P. 2004. MicroRNAs: Genomics, biogenesis, mechanism, and function. Cell 116: 281.

Bateman A. 2002. The SGS3 protein involved in PTGS finds a family. BMC Bioinformatics 3: 21.

Baumberger N. and Baulcombe D.C. 2005. Arabidopsis ARGONAUTE1 is an RNA slicer that selectively recruits microRNAs and short interfering RNAs. Proc. Natl. Acad. Sci. 102: 11928.

Bollman K.M., Aukerman M.J., Park M.Y. Hunter C., Berardini T.Z., and Poethig R.S. 2003. HASTY, the Arabidopsis ortholog of exportin 5/MSN5, regulates phase change and morphogenesis. Development 130: 1493.

Bowman J.L., Eshed Y., and Baum S.F. 2002. Establishment of polarity in angiosperm lateral organs. Trends Genet. 18: 134.

Chen X. 2004. A microRNA as a translational repressor of APETALA2 in Arabidopsis flower development. Science 303: 2022.

Dunoyer P., Himber C., and Voinnet O. 2005. DICER-LIKE 4 is required for RNA interference and produces the 21 -nucleotide small interfering RNA component of the plant cell-to-cell silencing signal. Nat. Genet. 37: 1356.

Emery J.F., Floyd S.K., Alvarez J., Eshed Y., Hawker N.P., Izhaki A., Baum S.F., and Bowman J.L. 2003. Radial patterning of Arabidopsis shoots by class III HD-ZIP and KANADI genes. Curr. Biol. 13: 1768.

Eshed Y., Baum S.F., Perea J.V., and Bowman J.L. 2001. Establishment of polarity in lateral organs of plants. Curr. Biol. 11: 1251.

Eshed Y., Izhaki A., Baum S.F., Floyd S.K., and Bowman J.L. 2004. Asymmetric leaf development and blade expansion in Arabidopsis are mediated by KANADI and YABBY activities. Development 131: 2997.

Fahlgren N., Montgomery T.A., Howell M.D., Allen E., Dvorak S.K., Alexander A.L., and Carrington J.C. 2006. Regulation of AUXIN RESPONSE FACTOR 3 by TAS3 ta-siRNA affects developmental timing and patterning in Arabidopsis. Curr. Biol. 16: 939.

Floyd S.K. and Bowman J.L. 2004. Gene regulation: Ancient microRNA target sequences in plants. Nature 428: 485.

Floyd S.K., Zalewski C.S., and Bowman J.L. 2006. Evolution of class III homeodomain-leucine zipper genes in streptophytes. Genetics 173: 373.

Garcia D., Collier S.A., Byrne M.E., and Martienssen R.A. 2006. Specification of leaf polarity in Arabidopsis via the trans-acting siRNA pathway. Curr. Biol. 16: 933.

Gasciolli V., Mallory A.C., Bartel D.P., and Vaucheret H. 2005. Partially redundant functions of Arabidopsis DICER-like enzymes and a role for DCL4 in producing trans-acting siRNAs. Curr. Biol. 15: 1494.

Grigg S.P., Canales C., Hay A., and Tsiantis M. 2005. SERRATE coordinates shoot meristem function and leaf axial patterning in Arabidopsis. Nature 437: 1022. 
Han J., Lee Y., Yeom K.H., Kim Y.K., Jin H., and Kim V.N. 2004. The Drosha-DGCR8 complex in primary microRNA processing. Genes Dev. 18: 3016.

Himber C., Dunoyer P., Moissiard G., Ritzenthaler C., and Voinnet O. 2003. Transitivity-dependent and -independent cell-to-cell movement of RNA silencing. EMBO J. 22: 4523.

Hunter C., Willmann M.R., Wu G., Yoshikawa M., de la Luz Gutierrez-Nava M., and Poethig S.R. 2006. Trans-acting siRNA-mediated repression of ETTIN and ARF4 regulates heteroblasty in Arabidopsis. Development 133: 297.

Jones-Rhoades M.W., Bartel D.P., and Bartel B. 2006. microRNAs and their regulatory roles in plants. Annu. Rev. Plant Biol. 57: 19.

Juarez M.T., Twigg R.W., and Timmermans M.C.P. 2004a. Specification of adaxial cell fate during maize leaf development. Development 131: 4533.

Juarez M.T., Kui J.S., Thomas J., Heller B.A., and Timmermans M.C.P. 2004b. microRNA-mediated repression of rolled leafl specifies maize leaf polarity. Nature 428: 84 .

Kerstetter R.A., Bollman K., Taylor R.A., Bomblies K., and Poethig R.S. 2001. KANADI regulates organ polarity in Arabidopsis. Nature 411: 706.

Kidner C.A. and Martienssen R.A. 2004. Spatially restricted microRNA directs leaf polarity through ARGONAUTE1. Nature 428: 81.

Kim J., Jung J.H., Reyes J.L., Kim Y.S., Kim S.Y., Chung K.S., Kim J.A., Lee M., Lee Y., Narry Kim V., et al. 2005. microRNA-directed cleavage of $A T H B 15$ mRNA regulates vascular development in Arabidopsis inflorescence stems. Plant J. 42: 84

Kumaran M.K., Bowman J.L., and Sundaresan V. 2002. YABBY polarity genes mediate the repression of $K N O X$ homeobox genes in Arabidopsis. Plant Cell 14: 2761.

Li H., Xu L., Wang H., Yuan Z. Cao X., Yang Z., Zhang D., Xu Y., and Huang H. 2005. The putative RNAdependent RNA polymerase RDR6 acts synergistically with ASYMMETRIC LEAVES1 and 2 to repress BREVIPEDICELLUS and MicroRNA165/166 in Arabidopsis leaf development. Plant Cell 17: 2157.

Mallory A.C. and Vaucheret H. 2006. Functions of microRNAs and related small RNAs in plants. Nat. Genet. 38: S31.

McConnell J.R., Emery J., Eshed Y., Bao N., Bowman J., and Barton M.K. 2001. Role of PHABULOSA and PHAVOLUTA in determining radial patterning in shoots. Nature 411: 709.

Otsuga D., DeGuzman B., Prigge M.J., Drews G.N., and Clark S.E. 2001. REVOLUTA regulates meristem initiation at lateral positions. Plant J. 25: 223.

Parizotto E.A., Dunoyer P., Rahm N., Himber C., and Voinnet O. 2004. In vivo investigation of the transcription, processing, endonucleolytic activity, and functional relevance of the spatial distribution of a plant miRNA. Genes Dev. 18: 2237.

Park W., Li J., Song R., Messing J., and Chen X. 2002. CARPEL FACTORY, a Dicer homolog, and HEN1, a novel protein, act in microRNA metabolism in Arabidopsis thaliana. Curr. Biol. 12: 1484 .

Pekker I., Alvarez J.P., and Eshed Y. 2005. Auxin response factors mediate Arabidopsis organ asymmetry via modulation of KANADI activity. Plant Cell 17: 2899.

Peragine A., Yoshikawa M., Wu G., Albrecht H.L., and Poethig R.S. 2004. SGS3 and SGS2/SDE1/RDR6 are required for juvenile development and the production of trans-acting siRNAs in Arabidopsis. Genes Dev. 18: 2368.

Reinhart B.J., Weinstein E.G., Rhoades M.W., Bartel B., and Bartel D.P. 2002. MicroRNAs in plants. Genes Dev. 16: 1616.
Rhoades M.W., Reinhart B.J., Lim L.P., Burge C.B., Bartel B., and Bartel D.P. 2002. Prediction of plant microRNA targets. Cell 110: 513.

Sawa S., Watanabe K., Goto K., Liu Y.G., Shibata D., Kanaya E., Morita E.H., and Okada K. 1999. FILAMENTOUS FLOWER, a meristem and organ identity gene of Arabidopsis, encodes a protein with a zinc finger and HMG-related domains. Genes Dev. 13: 1079.

Siegfried K.R., Eshed Y., Baum S.F., Otsuga D., Drews G.N., and Bowman J.L. 1999. Members of the YABBY gene family specify abaxial cell fate in Arabidopsis. Development 126: 4117.

Sussex I.M. 1951. Experiments on the cause of dorsiventrality in leaves. Nature 167: 651.

. 1955. Morphogenesis in Solanum tuberosum L.: Experimental investigation of leaf dorsiventrality and orientation in the juvenile shoot. Phytomorphology 5: 286.

Tang G., Reinhart B.J., Bartel D.P., and Zamore P.D. 2003. A biochemical framework for RNA silencing in plants. Genes Dev. 17: 49.

Timmermans M.C.P., Juarez M.T., and Phelps-Durr T.L. 2004. A conserved microRNA signal specifies leaf polarity. Cold Spring Harbor Symp. Quant. Biol. 69: 409.

Timmermans M.C.P., Schultes N.P., Jankovsky J.P., and Nelson T. 1998. Leafbladeless 1 is required for dorsoventrality of lateral organs in maize. Development 125: 2813.

Ulmasov T., Hagen G., and Guilfoyle T.J. 1999. Dimerization and DNA binding of auxin response factors. Plant J. 19: 309.

Vazquez F., Vaucheret H., Rajagopalan R., Lepers C., Gasciolli V., Mallory A.C., Hilbert J.L., Bartel D.P., and Crete P. 2004. Endogenous trans-acting siRNAs regulate the accumulation of Arabidopsis mRNAs. Mol Cell. 16: 69.

Vaucheret H. 2006. Post-transcriptional small RNA pathways in plants: Mechanisms and regulation. Genes Dev. 20: 759.

Vaucheret H., Vazquez F., Crete P., and Bartel D.P. 2004. The action of ARGONAUTE1 in the miRNA pathway and its regulation by the miRNA pathway are crucial for plant development. Genes Dev. 18: 1187.

Voinnet O. 2005. Non-cell autonomous RNA silencing. FEBS Lett. 579: 5858

Wardlaw C.W. 1940. Experiments on organogenesis in ferns. Growth (suppl.) 13: 93.

Williams L., Carles C.C., Osmont K.S., and Fletcher J.C. 2005a. A database analysis method identifies an endogenous trans-acting short-interfering RNA that targets the Arabidopsis ARF2, $A R F 3$, and ARF4 genes. Proc. Natl. Acad. Sci. 102: 9703.

Williams L., Grigg S.P., Xie M., Christensen S., and Fletcher J.C. 2005b. Regulation of Arabidopsis shoot apical meristem and lateral organ formation by microRNA miR166g and its AtHD-ZIP target genes. Development 132: 3657.

Xie Z., Allen E., Wilken A., and Carrington J.C. 2005. DICERLIKE 4 functions in trans-acting small interfering RNA biogenesis and vegetative phase change in Arabidopsis thaliana. Proc. Natl. Acad. Sci. 102: 12984.

Xu L., Yang L., Pi L., Liu Q., Ling Q., Wang H., Poethig R.S., and Huang H . 2006. Genetic interaction between the AS1AS2 and RDR6-SGS3-AGO7 pathways for leaf morphogenesis. Plant Cell Physiol. 47: 853.

Yang L., Liu Z., Lu F., Dong A., and Huang H. 2006. SERRATE is a novel nuclear regulator in primary microRNA processing in Arabidopsis. Plant J. 47: 841.

Yoshikawa M., Peragine A., Park M.Y., and Poethig R.S. 2005. A pathway for the biogenesis of trans-acting siRNAs in Arabidopsis. Genes Dev. 19: 2164.

Yu L., Yu X., Shen R., and He Y. 2005. HYL1 gene maintains venation and polarity of leaves. Planta 221: 231. 


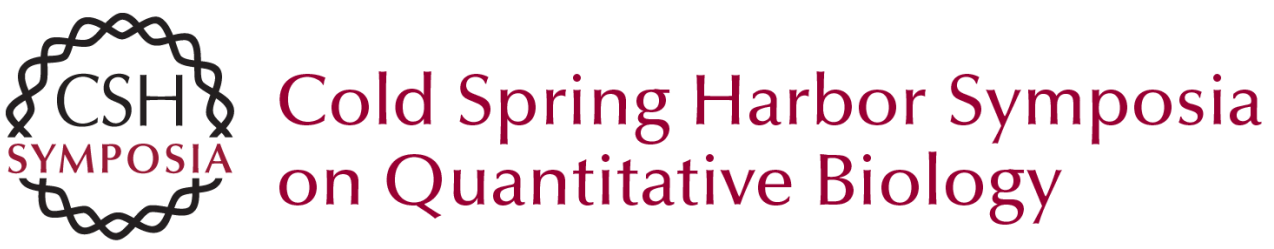

\section{Organ Polarity in Plants Is Specified through the Opposing Activity of Two Distinct Small Regulatory RNAs}

F.T.S. NOGUEIRA, A.K. SARKAR, D.H. CHITWOOD, et al.

Cold Spring Harb Symp Quant Biol 2006 71: 157-164

Access the most recent version at doi:10.1101/sqb.2006.71.045

References This article cites 60 articles, 27 of which can be accessed free at: http://symposium.cshlp.org/content/71/157.full.html\#ref-list-1

License

Email Alerting

Receive free email alerts when new articles cite this article - sign up in Service the box at the top right corner of the article or click here. 